Réplicas / Remarcks 


\section{Entre el mercado y la cooperación social. Luces y sombras de las prácticas de consumo colaborativo}

\section{Between the market and the social cooperation. Lights and shades of collaborative consumption practices}

\section{*Concepción Piñeiro}

Laboratorio de Socio-Ecosistemas. Departamento de Ecología. Universidad Autónoma de Madrid. España/Spain

Socia de Altekio Iniciativas hacia la Sostenibilidad S. Coop. Mad. España/Spain

conchi@altekio.es

\section{Rubén Suriñach}

Investigador y coordinador del área de consultoría de la cooperativa Opcions de Consum Responsable.

Coordinador del proyecto de Balance social de la Xarxa d'Economia Solidària

rubens@pangea.org

\section{José Luis (Kois) Fernández Casadevante}

Experto en soberanía alimentaria. Miembro de Garúa S. Coop. Mad. España/Spain

kois@garuacoop.es

\section{RESUMEN}

En el artículo se abordan diferentes definiciones del consumo colaborativo desde la teoría y la práctica. Para contextualizar el CC, se realiza una descripción de los elementos o marcos que sostienen su emergencia, estableciendo relaciones y diferencias con las otras economías transformadoras que están desarrollándose actualmente. A través de diferentes prácticas y ejemplos, se profundiza en las aportaciones del CC y en algunas de sus limitaciones, tales como las excesivas expectativas, la inseguridad jurídica, la mercantilización, la brecha digital, etc. En definitiva se muestra la contribución del CC a la crítica del sistema hegemónico capitalista y la generación de alternativas, evidenciando como al mismo tiempo su desarrollo puede representar tensiones en cuestiones como la ambiental-comunitaria, la regulación-desregulación, y la visibilidad de un paraguas común que aglutine a prácticas tan heterogéneas.

Palabras clave: Consumo Colaborativo, Economía Colaborativa, Consumo Transformador.

\section{ABSTRACT}

In this article different definitions of collaborative consumption (CC) are discussed from theory and practice. To contextualize the CC, there is a description of the elements or frames that support its emergence, establishing relationships and differences with other transformative economies that are currently being developed. Through different practices and examples, the contributions of CC are deepened and some of its limitations, such as excessive expectations, legal uncertainty, commodification, the digital divide, etc. In short it shows the contribution of CC as a critique of the capitalist hegemonic system and as part of the generation of alternatives and at the same time CC emphasizes paradoxes as the community-environment, the regulation - deregulation, and the vissibility of a common umbrella for so heterogeneous practices.

Keywords: Collaborative Consumption, Collaborative Economics, Transforming Consumption.

*Autor para correspondencia / Corresponding author: Concepción Piñeiro. Altekio. Calle Paseo de las Acacias, n. ${ }^{\circ}$ 3, 1. ${ }^{\circ}$ A. 28005 Madrid.

Sugerencia de cita / Suggested citation: Piñeiro, C., Surinach, R., Casadevante, J. L. (2017). Entre el mercado y la cooperación social. Luces y sombras de las prácticas de consumo colaborativo. Revista Española de Sociología, 26 (1), 97-108.

(http://dx.doi.org/10.22325/fes/res.2017.5) 


\section{¿QUÉ ES Y QUÉ NO ES EL CONSUMO COLABORATIVO? DIFERENTES DEFINICIONES DESDE LO ACADÉMICO Y DESDE LA PRÁCTICA}

El concepto Consumo Colaborativo (en adelante CC) lo acuñan por primera vez Bostman y Rogers (2010). Definen el CC como:

(...) la reinvención de los comportamientos de mercado tradicionales - alquilar, prestar, intercambiar, compartir, regalar- a través de la tecnología, tomando así formas y una escala imposible antes de internet.

En la misma publicación, definen tres tipos de sistemas de mediación para la satisfacción de necesidades: los sistemas basados en producto; los mercados de distribución; y los estilos de vida colaborativos. Los sistemas basados en producto serían pagar por el acceso a un producto sin la necesidad de adquirirlo, como por ejemplo alquilar coche 0 bicicletas para desplazarse. Los mercados de redistribución se basan en re-circular los bienes usados 0 adquiridos de donde ya no se necesitan hacia algún lugar 0 alguien que sí los necesita. El ejemplo más claro serían los mercados o redes de intercambio, y también los mercados de segunda mano. El tercero, los estilos de vida colaborativos, hace referencia a gente que se conecta entre sí para compartir e intercambiar bienes menos tangibles como tiempo, espacio, habilidades y dinero. Este último sistema es redefinido por un estudio elaborado conjuntamente por Ouishare y La Fing (2015), dividiéndolo en dos: por un lado los servicios de usuario a usuario (alquiler o intercambio entre personas individuales), y por otro los sistemas cooperativos locales (más vinculado a economías comunitarias basadas en las TIC o no necesariamente digitalizadas, como por ejemplo los grupos de consumo agroecológico).

En general, se considera que el CC es un subconjunto de la Economía Colaborativa (en adelante EC). Bostman (2015) la define así:

Un sistema económico compuesto de redes y ámbitos de intercambio descentralizados que liberan el valor de activos infrautilizados mediante el encuentro de demandas y ofertas, de un modo que no hacen falta intermediarios.
Lo que no está tan claro es cómo se subdivide 0 categoriza esta economía. Así, Bostman parte en gran medida del esquema circular de la renta de la economía clásica y dice que el consumo es uno de los ámbitos, pero que hay fórmulas colaborativas en las otras esferas del ciclo económico: financiación colaborativa a través de plataformas de micro-mecenazgo y producción colaborativa a través de infraestructuras y conocimiento compartido. Cañigueral (2014) añade un cuarto elemento: la producción abierta de conocimiento como base para todo lo demás. En cambio, Bauwens (2012) no parte tanto de las esferas del ciclo económico sino de qué se comparte 0 mutualiza: la producción y consumo de bienes (enfoque materiah o la producción y uso de conocimiento (enfoque inmateriah). En cualquier caso, la poca madurez de los debates conceptuales vendría a constatar que en esto de la EC las prácticas van por delante de los conceptos. Es desde esta mirada que la revista Opciones enfocó el número monográfico dedicado a EC:

Sabemos qué son, pero no sabemos muy bien cómo llamarlos: consumo colaborativo, innovaciones comunitarias, nuevas prácticas económicas, experiencias postcapitalistas, nowtopias... Es un saco que engloba nuevas y diversas prácticas económicas que están floreciendo, y que tienen en común algún grado de participación u organización colectiva en la provisión de bienes y servicios. Tratar de catalogarlas o clasificarlas es algo artificioso porque, como pasa en la mayoría de fenómenos reales, potentes e interesantes, las fronteras no son nítidas, ni falta que hace (Opciones, 2013).

A pesar de lo artificioso de intentar clasificar este fenómeno social, y con el fin de entender la emergencia y potencial transformador del mismo, sí que creemos que es conveniente, por lo menos, situar el CC en relación a dos grandes elementos. En primer lugar, creemos que es importante entender qué elementos de contexto explican la aparición del fenómeno y su crecimiento acelerado. En segundo, tratar de entender qué grado de 
desafío supone estas propuestas sobre las bases materiales y culturales del capitalismo, aportando las ventanas de oportunidad pero también los desafíos y peligros que enfrenta el CC.

\section{EMERGENCIA DEL CONSUMO COLABORATIVO Y SUS MARCOS DE ANÁLISIS}

Estos son algunos de los elementos o marcos que sostienen la emergencia del CC (basado en las fuentes consultadas en las referencias):

- Crisis del individualismo, y "re-descubrimiento" de lo comunitario. Como apunta Schor (2014), una de las motivaciones subyacentes en las personas que llevan a cabo prácticas de CC es la búsqueda de relaciones sociales y la construcción de comunidad. De hecho, el papel del CC en la construcción de lazos comunitarios es uno de los grandes lemas que abandera el movimiento de CC. Hay que tener en cuenta que, por su origen, es un movimiento que apela a un sentido de comunidad muy basado en el contexto cultural anglosajón, y el vacío existente en ese ámbito es común a los países occidentales (de larga implantación del capitalismo), y es por ello que su llamada a la necesidad de reconstruir los lazos comunitarios es reconocible desde países occidentales con diferencias culturales importantes. Uno de los grandes interrogantes es saber si las prácticas de CC dan respuesta a esa búsqueda de comunidad o no, si lo hacen en qué medida y qué tipo de comunidad desarrollan, etc.

- Conciencia ambiental. Otro de los marcos que acompaña el CC es el grado de reconocimiento de las problemáticas ambientales asociadas al modelo de producción, distribución y consumo por parte de la población. El CC es una forma palpable y "moderna" de dar una salida práctica a las inquietudes ambientales de la población. También en este caso, como plantea Schor (2014), es importante entender has- ta qué punto supone una reducción en el uso de recursos y el impacto ambiental.

- Las nuevas tecnologías y la sociedad en red. Otro gran elemento que explica la aparición y extensión del CC es el papel de las nuevas tecnologías de la información (TICS) y las dinámicas de sociedad en red que Ilevan asociadas. Así lo explican los impulsores del CC en España en su página web de referencia ${ }^{1}$ :

La eficiencia de Internet, combinada con la capacidad de crear confianza entre extraños, ha creado un mercado de intercambios eficientes entre productor y consumidor, prestador y prestatario, y entre vecino y vecino, sin intermediarios.

Aparecen así cuestiones propias como el acceso a la tecnología, así como interrogantes acerca de cómo este elemento se relaciona con los anteriores, la construcción de comunidad y la conciencia ambiental, tanto un nivel más filosófico del movimiento (por ejemplo en la lógica P2P, entre iguales o de usuaria a usuaria) qué tipo de comunidad propone, construye 0 promueve, como en su dimensión ambiental, qué impacto ambiental tienen las TICs y qué percepción hay de este impacto, y por extensión de otros impactos sociales de las TICs.

- Crisis económica y sobre-acumulación de patrimonio. Un cuarto elemento es la situación de necesidad económica y la existencia de patrimonio ocioso (Schor, 2014). EI CC abre la puerta a dar valor económico a capacidades 0 recursos a los que las formas de producción y consumo mayoritarias no daban cabida. En un contexto de profunda crisis económica y material, la posibilidad de obtener ingresos que abre el CC es pues un factor de atracción. También en estos casos se abren una serie de interrogantes

1 Extracto de la web Consumo colaborativo. Compartir reinventando la tecnología (en línea) http://www.consumocolaborativo.com/concepto/introduccion/, acceso 4 de noviembre de 2015. 
en torno a la mercantilización de aspectos de la vida que se han venido resolviendo desde las relaciones de reciprocidad, ayuda mutua y gratuidad, y, por tanto, sin mediación del dinero. Se sitúa así controvertidamente en el debate que también plantean los comunes, los cuales según Lafuente y Corsín (2015) no son patrimonializables, bien porque son inagotables 0 bien porque no son excluibles.

- Atracción por la novedad y por lo que es tendencia. Finalmente habría un quinto factor que explica la extensión del CC, que es su vinculación con grupos sociales altamente "digitalizados" y vinculados al desarrollo de nuevas tecnologías. Estos grupos sociales cuentan con una reputación dentro del marco cultural dominante que hace que la propuesta tenga mucha más capacidad de seducción y aparición en la esfera pública que si proviniera de otros grupos sociales.

Estos cinco aspectos y los interrogantes asociados tratan de explicar este fenómeno social, apuntando ya algunas de sus limitaciones 0 desafíos que implica, así como de las principales críticas o cuestionamientos que recibe el discurso y las prácticas de CC. Pero precisamente para entender mejor el contexto en el que emerge, hay una necesidad de situar este fenómeno en un marco más amplio donde se relaciona con otras lógicas económicas así como otras prácticas de consumo y estilos de vida basados en valores y criterios como la primacía de lo cercano y de lo local, la sostenibilidad, la justicia social, etc. 0 lo que Alonso et al. (2010) han Ilamado las formas de consumo alternativas y de autorresponsabilidad. Estas otras formas anteriores al término "consumo colaborativo" pueden verse gráficamente en la siguiente figura, destacando cómo en la recogida de datos realizada hasta 2010 el consumo colaborativo no aparecía en los discursos (Piñeiro, 2011);

Estas prácticas y marcos asociados preexistentes sostienen y se relacionan con el CC de múltiples formas. Porro (2015) ha hecho un primer esquema para situar las diferentes economías transformadoras que se relacionan con el consumo y los estilos de vida transformadores mencionados anteriormente, que hace visibles las relaciones entre la economía colaborativa y las otras economías en relación a dos criterios fundamentales que aparecen recurrentemente en los elementos citados que configuran los ejes: la reducción o minimización del impacto ecosocial (entendido como sus consecuencias perjudiciales a nivel social y ecológico) así como la distribución del poder y por extensión la generación de comunidad.

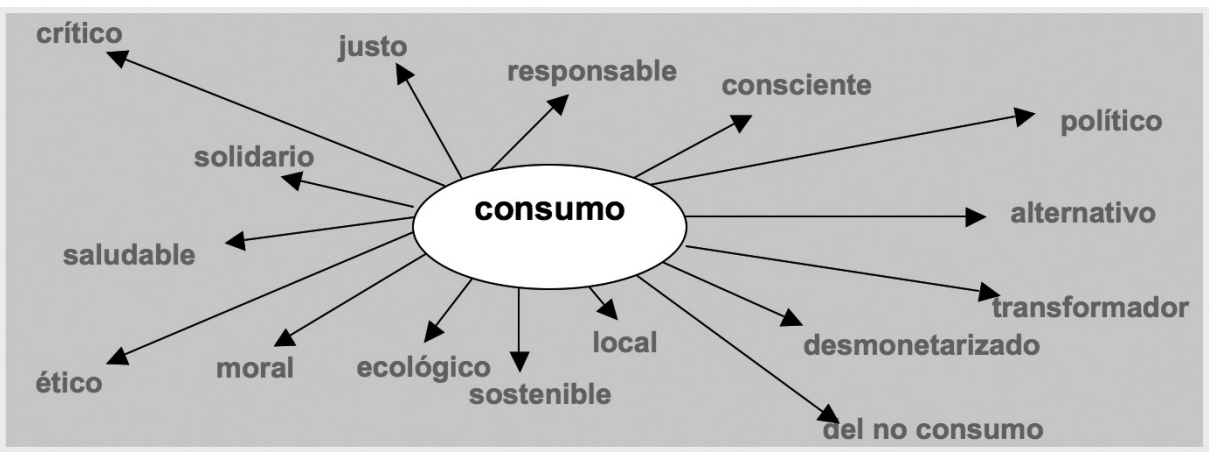

Figura 1. Etiquetas asociadas al tipo de consumo de autorresponsabilidad 


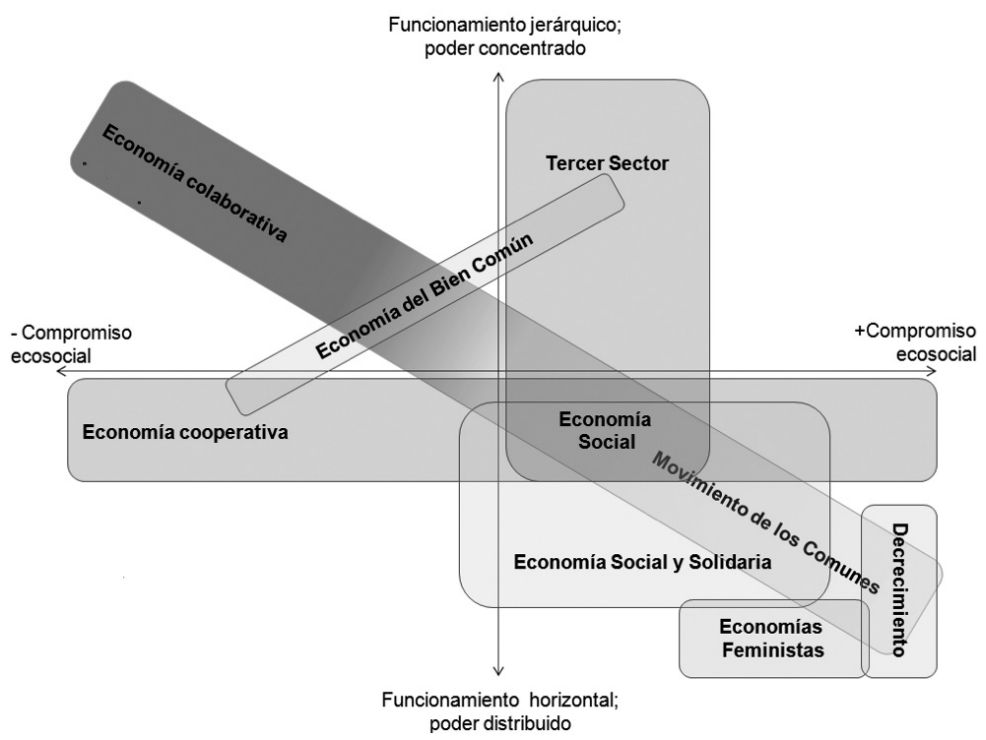

Figura 2. Mapa conceptual de las economías transformadoras

Fuente: Porro (2015)

Es difícil y puede resultar osado generalizar prácticas concretas y situadas para plasmarlas en esquemas generales, pero al mismo tiempo es necesario poner en el centro del debate los criterios implícitos en esas prácticas que a su vez generan conocimiento y tienen su propia forma de nombrarse.

De la necesidad de cambiar las formas de producción y consumo asociadas al capitalismo (en sus distintas fases históricas) han ido saliendo respuestas que intentan enfrentar uno, algunos 0 todos los cimientos sobre los que se asienta este marco dominante. Seguramente hoy, quizás como síntoma de las excesivas contradicciones del capitalismo, encontramos la mayor diversidad de propuestas económicas alternativas que se hayan visto en la historia reciente.

La economía social y el cooperativismo, por ejemplo, tienen una larga tradición y han construido su alteridad cuestionando la propiedad privada y capitalista de los medios de producción. Estas entidades y empresas funcionan dentro de la economía convencional, pero desconectadas de sus lógicas, valores y prácticas. Viables empresarialmente invierten, sin embargo, conscientemente, las priori- dades de la economía convencional: satisfacción de necesidades frente a rendimientos financieros, fuerte territorialización y vinculación con el entorno frente a la amenaza de deslocalización, promoción de procesos de cooperación frente a competencia, priorización de la rentabilidad social frente a la unidimensional tasa de ganancia, apuesta por el empleo y por los grupos sociales más vulnerables frente a aquellas intensivas únicamente en capital. Un movimiento que no ha parado de crecer durante los últimos años, y que se ha reafirmado en los principios solidarios, colectivos y ecológicos frente a derivas más mercantilistas, logrando introducirse en sectores estratégicos como el del acceso cooperativo a la banca, los servicios financieros, la producción y comercialización de energías renovables, los seguros, la alimentación o la vivienda.

Otro ejemplo son las prácticas y discursos sobre los comunes, que establecen sus marcos sobre las instituciones comunitarias de gestión de recursos estratégicos para comunidades locales estudiadas por Elinor 0strom, pero que se reactualizan con las nuevas tecnologías, los comunes digitales y como referencia crítica ante la crisis económica internacional. 
El CC, como los demás movimientos, nace del diagnóstico compartido de que hace falta transformar la manera en que las sociedades satisfacen sus necesidades, en este caso aquellas enriquecidas e intensivas en TICS). Esta nueva corriente se suma al río existente de economías alternativas que tiene la intención de "revolucionar" el marco dominante, cuestionando principalmente la manera en que las personas acceden a bienes y servicios, así como algunos aspectos de las formas de producción.

Seguramente hay otras economías que también deberían ser recogidas en la Figura 2 y su ubicación puede generar controversia. Precisamente lo que buscamos es abrir este diálogo entre las prácticas y los marcos, explicitando los ejes que son criterios en torno a los que podemos clasificarlas.

Por ejemplo, Del Moral y Fernández (2015) nos muestran cuatro escenarios distintos a partir del trabajo de la P2P Foundation (Kostakis \& Bauwens, 2014), mediante dos ejes diferentes. El primer eje describe la polaridad entre el control centralizado y el control distribuido de la infraestructura, frente a un segundo que muestra una orientación hacia la acumulación y circulación del capital, en contraste con una segunda orientación hacia la acumulación y circulación del procomún.

Surge así la EC en el marco de las transformaciones de la sociedad de consumo por la crisis del individualismo abriendo una nueva tercera vía en aspectos como la regulación o no regulación, y lo público o privado, que varía en cada experiencia concreta.

\section{HACIA UN DEBATE PRÁCTICO SOBRE LAS PRÁCTICAS}

En aras de profundizar en el entendimiento de algunas de las claves del CC hemos seleccionado algunas experiencias que explican su alcance. Para la selección, hemos utilizado la tipología descrita anteriormente (a partir de Bostman \& Rogers, 2010; Ouishare y La Fing, 2015), así como los criterios de ser experiencias en práctica en el territorio estatal, que cubren diferentes sectores o necesidades (transporte, alimentación, financiación, etc.) y con una diversidad en su origen (propuestas pro- movidas por colectivos o movimientos sociales de forma voluntaria, por un pequeño grupo promotor 0 personas en la búsqueda del autoempleo, empresas de distinta escala, etc.), apuntando así a la diversidad que suponen respecto a los debates mencionados en los distintos ejes citados en el marco (tipo de comunidad que genera y distribución del poder, origen y finalidad de la iniciativa, acumulación y circulación del capital/comunes, impacto ecosocial, etc.). De hecho esta diversidad en su origen es una de las cuestiones críticas, pues agrupa bajo un mismo término a iniciativas que debido a su origen, finalidad y forma de desarrollo son con 0 sin ánimo de lucro (tanto en la interacción como en la entidad que origina la iniciativa), lo que supone uno de los polos de tensión y controversia con la Economía Solidaria.

Partimos de la selección realizada por la revista Opciones para su número de Economías colaborativas y en la web www.consumocolaborativo.com, incorporando otros ejemplos:

- Los sistemas basados en producto, con sistemas como Social Car (socialcar.com), que cuenta con personas que ofrecen su vehículo en alquiler y es una S. L. fundada por empresarios del sector de las renovables inspirados en servicios similares en otros países, con una inversión inicial de un millón de euros ${ }^{2}$. En contraste por su modelo de gestión, estaría el Bicing, transporte público urbano basado en compartir bicicleta.

- Los mercados de redistribución, incluyen una gran diversidad de iniciativas respecto a los criterios mencionados. Por ejemplo, hay páginas webs de intercambio 0 de donación de objetos como nolotiro.org donde puedes participar sin necesidad de que haya un conocimiento mutuo 0 acuerdos construidos colectivamente, aunque sí que hay normas de funcionamiento y funciona gracias al trabajo de muchas personas que

2 Datos extraídos de la noticia Navas, J. A. (2012). Gane dinero alquilando su coche (en línea) http://www.elmundo.es/elmundo/2012/05/04/economia/1336136876. html, acceso 10 de noviembre de 2015 en: 
han desarrollado el software libre 3 . Y dentro de esta misma tipología, hay mercados 0 redes de intercambio/trueque más territorializadas como las de Catalunya (aglutinadas en http://intercanvis.net/Xarxes), donde la participación implica una mayor relación y construcción colectiva, contando con un origen arraigado en movimientos sociales catalanes. Hay mercados de intercambio especializados en sectores 0 necesidades como cosas infantiles (creciclando.com) 0 para material y libros escolares (www.truequebook.es). eBay formaría parte del extremo en el modelo de gestión empresarial, ya que se financia en la bolsa estadounidense, o la start-up Wallapop de origen barcelonés que ha recibido hasta 40 millones de financiación ${ }^{4}$.

- Los estilos de vida colaborativos, en los que se diferencian los servicios de usuario a usuario y los sistemas cooperativos locales. Considerando el primer tipo en términos de dinero, podríamos hablar de sistemas de micro-mecenazgo como Goteo, que además promueve la cultura libre y otros valores, que se diferencia de otras plataformas de micro-mecenazgo por este posicionamiento. Un extremo aquí sería AIRBNB, que facilita el proveer un servicio de alojamiento entre individuos. Respecto al segundo tipo, encontramos una gran diversidad en el ámbito de la alimentación, en los grupos o cooperativas de consumo, canales cortos de comercialización, numerosas formas de satisfacer la necesidad de alimentación contando con la participación de las personas en la construcción de ese sistema, no sólo con dinero, sino con tiempo, conocimiento, tareas específicas, etc. Encontramos desde cooperativas de producción que promueven este tipo de formas de consumo, apoyando la auto-or-

3 http://nolotiro.org/es/page/about

4 Datos extraídos de Novoa, J. (2015). Wallapop, la startup española de los 40 millones de dólares (en línea) http://marketing4ecommerce.net/wallapop-startup-40millones-dolares/, acceso 26 de abril de 2016. ganización de consumidoras/es, webs que fomentan esta organización (http://www. grupoagrupo.net/ 0 http://www.ecoagricultor.com/grupos-de-consumo/), hasta consumidores/as que se auto-gestionan y buscan diferentes productores/as para satisfacer algunas de sus necesidades, producción parcial de otros productos para satisfacer otras, 0 incluso entidades donde las funciones de producción y distribución son repartidas entre personas socias.

En el dossier de Economistas Sin Fronteras sobre "Economía en colaboración", aparecen como ejemplo el sistema LETS, la moneda social, Ios sistemas de trueque 0 bancos/intercambios de tiempo. Todos ellos son anteriores al término "consumo colaborativo" pero han sido re-significados dentro de este marco. Esta es una de las críticas posibles a este término CC que ha englobado prácticas anteriores basadas en diferentes criterios. Un reciente estudio (2016) coordinado por la Organización de Consumidores y Usuarios (OCU) muestra que las razones económicas (ahorro de dinero) o prácticas (facilidad de uso y flexibilidad de horario) son las más mencionadas entre las personas encuestadas usuarias, lo que cuestiona esta relación con otras iniciativas transformadoras.

EI CC, por sus múltiples efectos de atracción, ha re-significado muchas prácticas que se venían desarrollando bajo otros marcos y está entrando con fuerza en la economía alternativa, pero es Ilamativo también que haya llamado la atención a grupos financieros e instituciones que no cuestionan en ninguna medida el capitalismo global. Esto nos plantea varias preguntas acerca de las potencialidades y limitaciones del mismo.

\section{EXCESIVAS EXPECTATIVAS, INSEGURIDAD JURÍDICA, MERCANTILIZACIÓN, BRECHA DIGITAL Y OTRAS LIMITACIONES}

Históricamente de las innovaciones tecnológicas se han derivado profundos cambios socioculturales, que rápidamente han supuesto nuevos aprovechamientos económicos, dando lugar a un periodo durante el cual conviven de 
forma conflictiva viejas y emergentes formas de negocio. La formalización de las nuevas prácticas agrupadas bajo la noción de CC tampoco ha estado exenta de estas tensiones, donde se repiten las acusaciones de inseguridad jurídica, de pérdida de garantías para consumidores/as, de intrusismo y competencia desleal, de fiscalidad asimétrica, de empleos perdidos en los sectores afectados, etc. Una conflictividad que se ha centrado especialmente en las iniciativas que de forma coyuntural ocupan la agenda mediática 0 generan mayor alarma social (como UBER, BlaBlaCar, y AIRBNB) $)^{5}$.

La retórica sobre el CC ha enfatizado tanto las virtudes y potencialidades de estas nuevas formas de acceder a bienes y servicios que se ha llegado a divulgar como el surgimiento de un nuevo paradigma económico. Las excesivas expectativas puestas en estas prácticas se ilustran en las declaraciones de la revista Time (Walsh, 2011), cuando afirmaba que ésta es una de las diez ideas que cambiará el mundo: "una forma de arreglar los peores problemas, desde la guerra y la enfermedad hasta el paro y el déficit'. Una vez transcurridos unos años y con cierta perspectiva, sin negar los impactos culturales y económicos provocados, convendría redimensionar la capacidad transformadora de este fenómeno.

Más allá de las estrategias individuales para satisfacer necesidades cotidianas, el CC se va configurando, ante la desorientada mirada de la economía tradicional, como un espacio de disputa. Asistimos a la difícil convivencia entre quienes lo conciben como una herramienta que permite la adaptación del mercado a los usos sociales de las nuevas tecnologías (innovación empresarial, nuevas fórmulas de negocio basadas en la interacción, la flexibilidad, la instantaneidad, la personalización...) y quienes persiguen una democratización extensa de las relaciones económicas y profundos cambios culturales (expectativas, deseos, patrones de consumo...).

5 Movilizaciones vecinales en Barcelona contra los problemas de convivencia derivados del auge de pisos turísticos, movilizaciones y denuncias del gremio del taxi hacia UBER, denuncia y espías contratados por la patronal de los autobuses contra BlaBlaCar.
El CC puede fomentar una suerte de capitalismo netárquico (Bauwens, 2013), donde se tiende a la concentración en grandes plataformas, que facilitan la accesibilidad, pero a cambio de acaparar el control e imponer lógicas empresariales. Muchas de estas grandes plataformas se encuentran financiadas por fondos de inversión que exigen una rápida rentabilidad económica, habiéndose dado casos de una fuerte precarización en los derechos laborales de su personal empleado. Una dinámica donde la dimensión social se diluye y se tiende a conformar comunidades de usuarios/as con vínculos extremadamente frágiles y procesos de cooperación restringidos, reduciéndose a interacciones ocasionales donde lo relacional es un mero peaje para acceder a intereses individuales. 0 incluso donde las personas que dependen de estas plataformas para generar ingresos carecen totalmente de derechos laborales, por lo que puede ser una forma de trabajo totalmente desprotegida. La conservadora web de noticias norteamericana Human Events, hizo este elogio de la economía del compartir: "Es el punto final del cada vez más caro y fuertemente regulado asunto de contratar trabajadores. A medida que la carga laboral se incrementa, unos acuerdos tarifa-por-servicio creativos resultan alternativas atractivas a los caros y tradicionales puestos de trabajo. Este sería el siguiente paso lógico después de la transición a gran escala de la fuerza laboral norteamericana hacia los puestos de trabajo a tiempo parcial (Bulajewski, 2014).

El CC suponía una excusa para compartir y reconstruir lazo social, un complemento para economías precarias que obtenían ingresos extras y la posibilidad de desmercantilizar la satisfacción de algunas necesidades. Sin embargo al no movilizar de forma consciente un imaginario crítico, puede terminar fomentando un consumismo colaborativo (Calle y Fdez. Casadevante, 2015) que mantenga nuestra dependencia de nuevas formas de mercado y nos lleve a interiorizar la necesidad de maximizar la rentabilidad económica de todo lo que tenemos. Además existe el riesgo de que estas prácticas al popularizarse aumenten los impactos ambientales, en vez de reducirlos, como demuestra repetidamente el efecto rebote o paradoja de Jevons (los ahorros generados por la eficiencia tecnológica son menores de lo que supone la popularización de los consumos). 
Tabla 1. Comparación entre el consumo colaborativo transformador y el capitalismo netárquico

\begin{tabular}{l|l}
\hline Consumo colaborativo transformador & Capitalismo netárquico \\
\hline El cambio social es intencional & $\begin{array}{l}\text { El cambio social es una consecuencia derivada no } \\
\text { perseguida conscientemente }\end{array}$ \\
\hline Desmercantilización en la satisfacción de necesidades & Mercantilización de la vida cotidiana \\
\hline Intensificar vínculos sociales y comunitarios & $\begin{array}{l}\text { Interacción social como peaje a pagar por acceder a } \\
\text { bienes y servicios }\end{array}$ \\
\hline Impulsar nuevos patrones de consumo consciente y & Consumismo colaborativo \\
\hline transformador & Plataformas cerradas, propietarias y centralizadas \\
\hline Territorialización, descentralización y código abierto a economía solidaria y lógica de los comunes & Nuevo nicho de mercado dentro de la economía capitalista \\
\hline Protagonismo ciudadano & Acumulación de capital a través de la cooperación \\
\hline Finanzas éticas y crowdfounding & Fondos de inversión capital riesgo \\
\hline
\end{tabular}

Fuente: Elaboración propia

La ambivalencia del consumo colaborativo se muestra en la Tabla 1, evidenciando como su desarrollo puede ser una herramienta al servicio de la transformación social 0 una simple adaptación del mercado a los nuevos ecosistemas de producción y consumo facilitados por las nuevas tecnologías. En el trabajo coordinado por la OCU en 4 países europeos se demostraba que solamente $1 / 4$ de las plataformas de consumo colaborativo estaban orientadas a la comunidad y perseguían explícitamente cambios sociales (OCU, 2016). Las variables principales en torno a las que se define el papel que juegan las distintas plataformas serían: la intencionalidad del cambio o no, la desmercantilización o no de la satisfacción de necesidades, el fomento de nuevas expectativas y valores en torno al consumo; el tamaño, la propiedad y la centralización de las plataformas o los mecanismos de financiación de las mismas.

\section{REFLEXIONES FINALES}

El CC contribuye a la crítica del sistema hegemónico capitalista y a la generación de propuestas, a partir de la inteligencia colectiva, por lo que ge- nera conocimientos y nuevos contextos necesarios para los desafíos actuales del Cambio Global. Pero al mismo tiempo el CC acentúa paradojas como la ambiental y la comunitaria, o tensiona la cuerda entre la regulación y la desregulación, evidenciando que en su interior hay tanta diversidad que es necesario diferenciar y separar una serie de prácticas de otras.

El CC ha asimilado prácticas que preexistían 0 que proceden de otros marcos de referencia como la economía solidaria o la agroecología, que ahora son incluidas y agrupadas dentro de estas propuestas. Resulta tentador aglutinar experiencias tan heterogéneas para analizarlas, entenderlas y contextualizarlas, aunque eso implique problematizar los conceptos unificadores como hemos realizado en este artículo.

EI CC se convierte en un espacio de disputa entre su dimensión transformadora, una pieza clave dentro de un puzzle más complejo que persigue la transformación social, conectándolo con la lógica de los comunes y la economía solidaria, y la que lo concibe como una mera adaptación de los mecanismos de mercado a un contexto modificado por las nuevas tecnologías. Ahora que el desarrollo del CC empieza a mostrar limitaciones que lo alejan 
del idealismo con el que fue abordado inicialmente, se muestra como un espacio de encuentro, dialogo, complicidad y confrontación donde durante las próximas décadas están condenadas a coexistir y convivir de forma conflictiva distintas formas de concebir la economía.

\section{REFERENCIAS BIBLIOGRÁFICAS}

Alonso, L. E., Fernández, C.; Ibáñez, R. y Piñeiro, C. (2010). Proyecto de investigación en consumo y estilos de vida. Informe de resultados. Manuscrito no publicado. Universidad Autónoma de Madrid.

Bauwens, M. et al., (2012). Syntethic overview of the Collaborative Economy (en línea) http:// p2pfoundation.net/Synthetic_Overview_of_ the_Collaborative_Economy, acceso 15 de noviembre de 2015.

Bauwens, M. (2013). Cuatro escenarios futuros para la economía colaborativa (en línea) http:// www.guerrillatranslation.es/2013/06/06/cuatro-escenarios-futuros-para-la-economia-colaborativa/, acceso 15 de noviembre de 2015.

Botsman, R. y Rogers, R. (2010). What's Mine Is Yours: The Rise of Collaborative Consumption, New York: Harper Business.

Bostman, R. (2015) Thinking. (en línea) http:// rachelbotsman.com/thinking/, acceso 4 de noviembre de 2015.

Bulajewski, M. The cult of sharing (en línea) mrteacup.org, acceso 5 de agosto de 2014.

Calle, A. y Fdez. Casadevante, J. L. (2015). Economías sociales y economías para los Bienes Comunes. Revista Otra Economía, 9. Brasil.

Cañigueral, A. (2014). Vivir mejor con menos: Descubre las ventajas de la nueva economía colaborativa. España: Conecta.
Del Moral, L. y Fernández, M. (2015). ¿Ha llegado para quedarse? Economía colaborativa, ciudad y universidades andaluzas. (en línea) http://www.academia.edu/11339032/_Ha_ Ilegado_para_quedarse_Universidad_y_ Econom\%C3\%ADa_Colaborativa, acceso 4 de noviembre de 2015.

Economías Colaborativas. Cuaderno central. 2013. Revista Opciones, 44. http://revistaopciones. org/es/revista/44-economias-colaborativas.

Economía en colaboración. 2014. Dossier n. ${ }^{0} 12$ de Economistas Sin Fronteras.

Lafuente, A. y Corsín, A. (2015). Economías y tecnologías del don. El procomún y los bienes comunes. Dossieres EsF. n. ${ }^{0} 16$, invierno de 2015.

Ouishare y La Fing (2015). Share revolution is now available in English (en línea) http://magazine.ouishare.net/2015/04/sharevolution-is-now-available-inenglish/, acceso 23 de noviembre de 2015.

Organización de Consumidores y Usuarios (coord.) (2016). Collaboration or business? Collaborative consumption: from value for users to a society with values. España: OCU Ediciones, S. A.

Piñeiro, C. (2011). Comunicación ambiental para la transformación social. Iniciativas de consumo responsable en Madrid. Tesis doctoral. UAM.

Porro, A. (2015). Presentación acerca de las economías comunitarias y grassrrots innovations. Switchmed Connect. Barcelona. Documento con licencia Creative Commons.

Schor, J. (2014). Debating the sharing economy (en línea) http://www.greattransition.org/publication/debating-the-sharing-economy, acceso 12 de noviembre de 2015.

Walsh, B. (2011). Today's Smart Choice: Don't Own. Share (en línea) http://content. time.com/time/specials/packages/article/0,28804,2059521_2059717_2059710,00. html, acceso 16 noviembre de 2015. 